UDC 004.738.5:316.77

Survey Paper

\title{
A Study on Recommendation Systems in Location Based Social Networking
}

\author{
Lakshmi Shree Kullappa \\ Faculty of Information Science and Engineering \\ Vemana Institute of Technology \\ Bangalore, India
}

Ranganath Ashok Kumar

Faculty of Information Science and Engineering

BMS College of Engineering,

\section{Rajeshwari Kullappa}

Faculty of Information Science and Engineering BMS College of Engineering

lakshmishreek@gmail.com

\author{
ashokkumar.ise@bmsce.ac.in
}

rajeshwarik.ise@bmsce.ac.in

\begin{abstract}
Smart devices in the hands of people are revolutionizing the social lifestyle of one's self. Everyone across the world are using smart devices linked to their social networking activities one such activity is to share location data by uploading the tagged media content like photos, videos. The data is of surroundings, events attended/attending and travel experiences. Users share their experiences at a given location through localization techniques. Using such data from social networks an attempt is made to analyze tagged media content to acquire information on user context, individual's interests, tastes, behaviors and derive meaningful relationships amongst them are referred to as Location Based Social Networks (LBSNs). The resulting information can be used to market a product and to improve business, as well recommend a travel and plan an itinerary. This paper presents a comprehensive survey of recommended systems for LBSNs covering the concepts of LBSNs, terminologies of LBSN and various recommendation systems.
\end{abstract}

Keywords: GPS trajectories, user similarity, Location based social networks, Collaborative Filtering, Recommendation systems.

\section{Introduction}

A social network is a social structure made up of individuals connected by one or more specific types of interdependency such as friendship, common interests, and shared knowledge. In addition, it can be described as a digital representation of the real world permitting users to share events, interests, likes/dislikes, ideas, and activities. 
The social networking services can be enhanced through Location Based Social Networks (LBSNs) as users share their location data. Location Based Social Networks are defined as a social structure made up of individuals connected by the interdependency derived from their locations in the physical world as well as their location-tagged media content such as photos, videos, and texts [1].

Various terminologies in LBSN are: -

i. Trajectories - Captures the physical movements and patterns of users in the real world obtained from the GPS (Global Positioning System) data. It typically consists of a sequence of spatio-temporal points in the form of the latitude, longitude and the time.

ii. GPS trajectory - A sequence of time-stamped latitude/longitude pairs, which are collected in every few seconds.

iii. Stay points - Stay points as shown In Figure 1 are the geographic regions or locations at which a user has spent sufficiently longer time. These locations are identified from the GPS log and can be treated as locations.

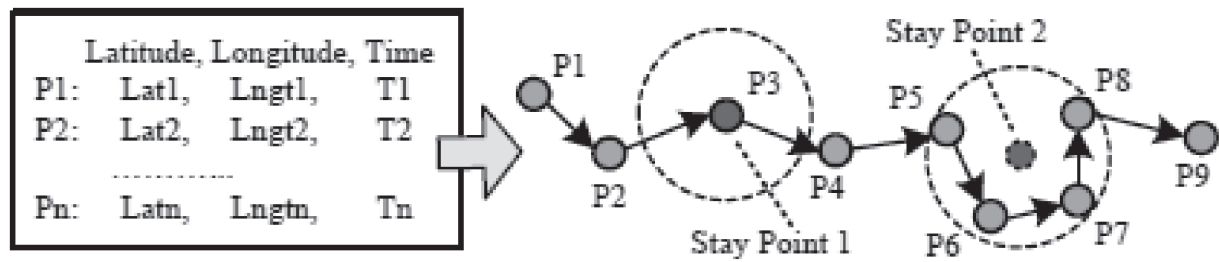

Figure 1: Representation of the GPS trajectories [1] in the form of Latitude / Longitude of the Stay Points from the GPS trajectories

iv. Location history - A location history is a record of location's that a user has visited in geographical spaces over a period.

v. Similar users - Two users who co-occur in the same physical location, share similar locations histories, interests, behavior activities and have same level of knowledge about a location. Similar users are inferred from an individual's location / location history and location-tagged data.

\subsection{Location Based Social Networks: The Concepts}

A Location $\left(L_{i}\right)$ is an object with dimension acquired from the users, who dynamically move to a sequence of locations $L_{1}, L_{2}, L_{3}, \ldots L_{n}$ which can either be outdoor or indoor environments. The preliminary concepts of LBSN are briefly described In Table 1 [3]. Each location is represented in various forms of which firstly we have absolute indicating latitude-longitude coordinates, secondly relative indicating approximate value, for instance $1 \mathrm{~km}$ south of a place $X$ and lastly symbolic represents home, office or shopping mall. A location-based social networking service is classified as a point location - based service where individuals share their exact location (office, restaurants), geo - tagged media based service are locations organized based on the 
media (videos, photos on social media) and lastly trajectory based location service provides initial and destination points of users (Microsoft GeoLife service).

\begin{tabular}{|l|l|l|l|}
\hline $\begin{array}{l}\text { Location } \\
\text { Acquisition } \\
\text { Technology }\end{array}$ & $\begin{array}{c}\text { Outdoor: Using } \\
\text { GPS mobile devices } \\
\text { (GSM/CDMA) }\end{array}$ & \multicolumn{2}{|c|}{$\begin{array}{l}\text { Indoor: Using Wi-Fi ,RFID, } \\
\text { Supersonic }\end{array}$} \\
\hline $\begin{array}{l}\text { Representation } \\
\text { of Locations }\end{array}$ & $\begin{array}{l}\text { Absolute (Latitude- } \\
\text { Longitude co- } \\
\text { ordinates) }\end{array}$ & Relative & Symbolic \\
\hline $\begin{array}{l}\text { Forms of } \\
\text { Locations }\end{array}$ & Point locations & Regions & Trajectories \\
\hline Services & Geo--Tagged - & $\begin{array}{l}\text { Point - Location } \\
\text { driven }\end{array}$ & $\begin{array}{l}\text { Trajectory - } \\
\text { Centric }\end{array}$ \\
\hline Properties & Hierarchical & $\begin{array}{l}\text { Sequential } \\
\text { Ordering }\end{array}$ & $\begin{array}{l}\text { Measurable } \\
\text { Distance }\end{array}$ \\
\hline
\end{tabular}

Table 1: LBSN concepts

In LBSN, inclusion of user's and their locations creates relationships between users and the locations among users can be represented as graphs $G(N, E)$ where $N$ is a node, E is an edge, N, E varies differently among graphs as shown In Figure 2. Association among users and locations is represented as user-graph, location-graph and user-location graph. In each graph, there are two types of nodes - user and location and three kinds of links - user-user, location-location, and user-location. As a user visits a series of locations in the physical world, a sequence of locations is created. The sequence is obtained from the individual's geo-data such as GPS trajectories, check-in records, and location based media. Geo-tagged-media-based service enables users to label the media content such as text, photos and videos generated in the physical world, to add location details either explicitly or implicitly when the content is created. Comparing to user's check-in data, GPS data is much denser and contains more routine activities (e.g., travelling from home to office every morning).

A LBSN's block diagram is shown In Figure 3. The processing of user's information

can be achieved using recommendation algorithms. Various recommendation algorithms and techniques used in the LBSN are explained in the Section 3. 


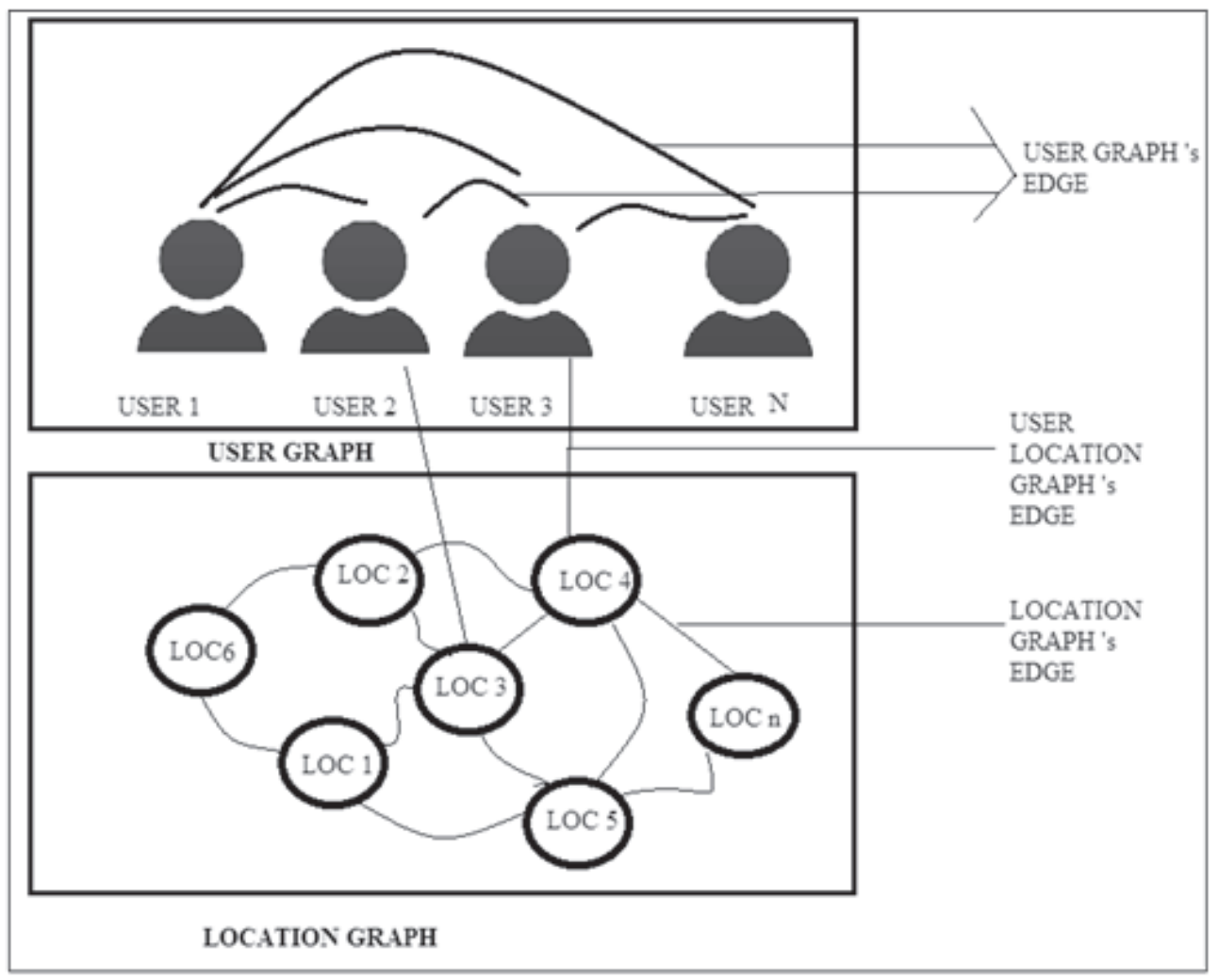

USER LOCATION GRAPH

Figure 2: Location Based Social Networks - Concept

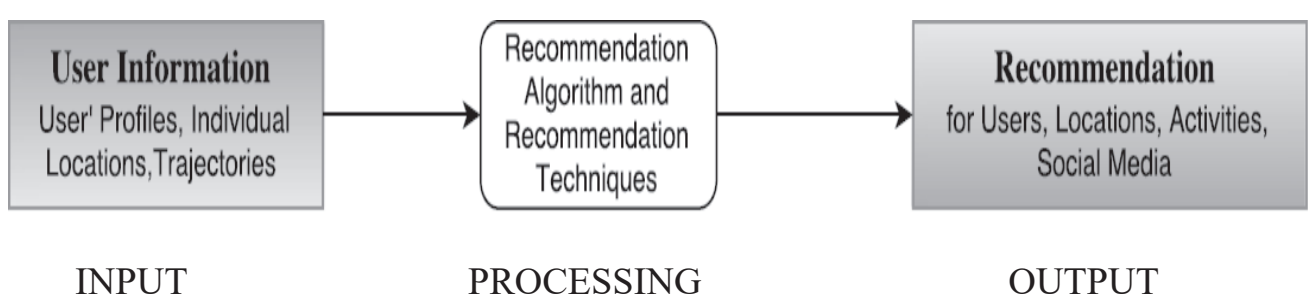

Figure 3: Block diagram for a LBSN

\subsection{Unique Properties of Locations}

Each location has few unique properties which describe its characteristics. Such as

i. Hierarchical - The location description can be described in various granularity levels like finer and coarse. Example: "A user dines at restaurant" and "A user dines at restaurant in a city" are the coarse and finer levels respectively. 
ii. Measurable Distances - Describes distance among user's location, distance between a user and his location and the distance between two locations.

iii. Sequential ordering - Describes the pattern in which the user visits the places and their timestamps. These details can provide the similarities between users, their preferences and interests.

\section{Recommendation System}

\subsection{The Role of the Recommendation Systems in LBSN}

Individuals around the world are relying on search engines for responses on numerous options like buying a product either through an online retailer or from stores, to watch a movie, visit a popular place (like a park, museum, or a hotel nearby). As individuals come across with many options, choosing amongst them becomes hard. Though there are numerous search engines to provide the details, results provided are not customized to individual's tastes as they are query-dependent. Individuals prefer to obtain the results, which are based on his/her preferences and personal choices to decide easily, quickly, and more accurately. Using recommender systems, this can be resolved.

\begin{tabular}{|l|c|}
\hline $\begin{array}{c}\text { Location } \\
\text { Recommendation }\end{array}$ & \multicolumn{1}{c|}{ Data Source } \\
\hline Stand Alone & $>$ User Profile based \\
& $>$ Location Histories \\
& $>$ User Trajectories \\
\hline Sequential & $>$ Geo-Tagged Social Media \\
& $>$ User GPS Trajectories \\
\hline
\end{tabular}

Table 2: Data Sources for various recommendation systems

There are many applications and algorithms designed for recommender systems, which can recommend individuals based on their choices. Recommendation algorithm is a function $(F)$ which is a product of a set of users $(U)$ and set of objects (I) [products/locations]. When a user queries for a best hotel in the town, recommendation systems employs browsing history, product purchases, user profile information and friend's information to make customized recommendations and returns different recommendations based on personal choices. Information such as social context, friendship, ratings of the user's and locations are the valuable 
information for the recommendation algorithm. The Location Recommendations and its data sources are listed In Table 2.

\subsection{The Classical Recommendation Algorithms}

The algorithms [4] can be classified as

i. Content Based Algorithms -The similarity between location's description and the user's profile information is measured. Higher the similarity, higher is the chance of the location being recommended. A mapping of the user preference with the location description is prepared where the users can pose questions to obtain similar results wherein inputs are location features and descriptions.

The results are based on the user's preferences, user profiles and contextual parameters. A Cosine similarity is used to measure the similarity.

ii.Collaborative Filtering $(\mathrm{CF})$ - User's preferences or the chances to visit a location increases when the locations are recommended by similar users. A user-location matrix is generated by using user profiles (information about the user's tastes, preferences), contextual parameters and community data behave as the input to the recommendation system. The users then pose questions for popular /recommended locations. Each user-location matrix entry is the ratings of the users to a specific location. These entries either are given or are missing. The techniques such as Clustering, Matrix Factorization and dimensionality reduction techniques such as Singular Value Decomposition (SVD) are used. Similarity can be measured by Pearson and Cosine similarity.

a. Memory Based Collaborative Filtering - In the user-location matrix, there can be few missing ratings. To fill these missing ratings, predictions are made considering the previous ratings of the location given by users who had already visited that location. Two Collaborative Filtering methods of Memory Based are User Based and Location Based.

- User Based Collaborative Filtering - User's ratings given in the past for locations are taken into consideration for rating next location to be visited. To remove noise and reduce computation number, the number of similar users can be limited to some fixed size say $(\mathrm{N})$ (limited to some positive integer). Among top $\mathrm{N}$ similar user's ratings are taken into consideration.

- Locations Based Collaborative Filtering - Locations with similar ratings from users in the past are taken into consideration for predicting the future users. For the similarity computation an average rating of the different users is considered.

b. Model Based Collaborative Filtering - Here the underlying model /hypothesis is learnt to predict the missing ratings in the User - location Matrix. Various techniques [4] used are

- $\quad S V D$ - This is a linear algebra technique with two matrices and the product of these two matrices is equivalent to the original matrix indicating a Lossless Matrix Factorization thereby yielding to lossless information.

- Eckart-Young-Mirsky Low-Rank Matrix Approximation - This is a best lowrank matrix approximation applied to remove noise. The matrix generated is not randomly generated but has an underlying structure. 
Other processes used in the Collaborative Filtering (CF) Recommendations are

- Candidate Selection - A subset of candidate nodes is selected to reduce computational overhead. Selection is based on geographical bounds, other association's constraints and on user preferences / social influences.

- Similarity influences - An analysis on various patterns of the locations visited is prepared. The similarity is inferred from the user ratings, interests, preferences, demographic data, spatial search logs, user interaction patterns (user tags and the comments shared with online friends of the location visited), location histories obtained from check-ins and feedbacks of the visitors.

- Recommendation Score Prédiction - A Recommendation Score of the location is calculated from the candidate sets to find the top- $k$ users who are said to be more similar.

iv. Link Recommendations - The recommendation system is based on the link analysis of a complex network say $\mathrm{G}(\mathrm{V}, \mathrm{E})$ where the $\mathrm{V}$ is set of vertices and the $E$ is set of Edges. Each edge also called as link is created between the vertices that are related. For instance, friendship between users creates a link. Link predictions, predicts an edge for a pair of nodes in some predefined training period by extracting high quality / influential nodes. The recommendation prefers experienced users and popular locations. Recommendations is based on assigning scores to the network. Further these scores are arranged in decreasing order in a testing interval of time from which top $N$ scores is derived and considered for recommendation. Scores can be classified as hub score (indicates the most number of users who have visited locations) and popularity score (indicates a location assessed by more number of users). The approaches used are PageRank, RandomWalk, and Hyperlink-Induced Topic Search (HITS).

iv. Hybrid Recommendation - Recommendation is a combination of Content based recommendation and Collaborative filtering recommendation. Combination is either in parallel or cascading manner. This technique improves the recommendation by combining the advantages of these techniques and overlook their individual disadvantages. User profiles, ratings, contextual parameters, product features, knowledge models and community data act as the input to the recommendation system.

iv. Knowledge-based Recommendation - In this recommendation system, for each user the knowledge about users, location's that is interesting and preferable are collected. Based on this information, a brief reasoning about the locations, which meets the user's tastes, is generated. Recommendations can be either Constraint or Case based. In constraint based, an explicitly defined set of recommendation rules are used to accomplish recommendation. These constraints depend on current user model attributes like the user features and location model. Whereas, in Case based, the location recommendation is based on the interests towards locations and results of similar locations is retrieved. 


\begin{tabular}{|l|l|}
\hline \multicolumn{1}{|c|}{ RS } & \multicolumn{1}{c|}{ TECHNIQUE } \\
\hline Content Based & $>$ Bayesian Classifiers \\
& $>$ Clustering \\
& $>$ Decision Tree \\
& $>$ TF-ID for Information Retrieval \\
\hline Collaborative Filtering & $>$ Nearest Neighbor Cosine Similarity \\
& $>$ Clustering \\
& $>$ Graph Theory \\
& $>$ Bayesian Classifiers \\
& $>$ Probabilistic Models \\
\hline Link Recommendation & $>$ Linear Combination Predicted Model \\
& $>$ Voting Schemes \\
\hline
\end{tabular}

Table 3: A summary of the recommendations techniques

\begin{tabular}{|l|l|l|}
\hline RS & \multicolumn{1}{|c|}{ Pros } & \multicolumn{1}{c|}{ Cons } \\
\hline 1 & $>\begin{array}{l}\text { Robust against the cold start } \\
\text { problem. } \\
\text { RS requires structured } \\
\text { information of users and } \\
\text { locations }\end{array}$ & $>\begin{array}{l}\text { Does not consider the } \\
\text { aggregated community } \\
\text { opinions }\end{array}$ \\
& $>\begin{array}{l}\text { Neywords alone may not be } \\
\text { Nufficient to judge } \\
\end{array}$ & $\begin{array}{l}\text { quality/relevance of an } \\
\text { interested document or web } \\
\text { page }\end{array}$ \\
\hline
\end{tabular}




\begin{tabular}{|c|c|c|}
\hline 2 & $\begin{aligned}> & \text { Supports Personalized } \\
& \text { recommendations } \\
> & \text { Quality is improved over time. } \\
> & \text { The knowledge of } \\
& \text { product is not required. }\end{aligned}$ & $\begin{aligned}> & \text { Has Data sparsity } \\
> & \text { Requires larger number of user } \\
& \text { ratings. } \\
> & \text { Requires additional information } \\
& \text { (user community) } \\
> & \text { Suffers from cold start and data } \\
& \text { sparsity problem }\end{aligned}$ \\
\hline 3 & $\begin{array}{l}\text { Robust against the cold start } \\
\text { problem. } \\
>\text { Supports personalized } \\
\text { recommendations (quality is } \\
\text { improved over time). }\end{array}$ & $\begin{array}{l}>\text { Requires Domain knowledge } \\
>\text { Sensitive to preferences } \\
\text { changes }\end{array}$ \\
\hline 4 & $\begin{array}{l}>\text { Robust against the cold start } \\
\text { problem } \\
>\text { User rating based on experience } \\
\text { of users on a location. }\end{array}$ & $\begin{array}{l}>\text { Does not consider the } \\
\text { personal. }\end{array}$ \\
\hline 5 & $\begin{array}{l}>\text { Solves the cold start problem } \\
>\text { Recommendations does not } \\
\text { depend on user rather on the } \\
\text { qualitative preference feedback } \\
>\text { Does not have to gather } \\
\text { information about a user only } \\
\text { the tastes are superior } \\
>\text { Sensitive to preferences } \\
\text { changes }\end{array}$ & $\begin{aligned} & \text { Taste /Interest are not a static } \\
& \text { parameter } \\
& \text { Need for Knowledge } \\
& \text { engineering. }\end{aligned}$ \\
\hline
\end{tabular}

Table 4: A summary of the recommendations algorithms

In Table 3, we discuss various techniques used in each of RS is represented. And In Table 4, a comparison of different recommendations systems (RS) is discussed with the pros, cons.

\subsection{Evaluation of Recommendations}

Recommendation algorithms [4] can be evaluated based on various criteria's is described.

a. Mean Absolute Error (MAE) - Computes the average absolute difference between the predicted ratings and true ratings.

Formulated as

$$
M A E=\frac{\sum_{i j}\left|\hat{r}_{i j}-r_{i j}\right|}{n}
$$


Where $n$ is the number of predicted ratings, $\hat{\boldsymbol{r}}_{\boldsymbol{i}}$ is the predicted rating and $\boldsymbol{r}_{\boldsymbol{i}}$ is the true rating.

b. Normalized Mean Absolute Error (NMAE) - Normalizes Mean Absolute Error by dividing it by the range ratings.

Formulated as

$$
\mathrm{NMAE}=\frac{\mathrm{MAE}}{\mathrm{r}_{\max }-\mathrm{r}_{\min }}
$$

Where $r_{\max }$ is the maximum rating and $r_{\min }$ is the minimum rating.

c. Root Mean Square Error (RMSE) - Measure of the differences between ratings predicted by a model and the ratings actually observed. RMSE is illustrated as

$\frac{\text { Good locations recommended }}{\text { All Good locations recommendation. }}$

Various measures for evaluation of the recommendation systems are: -

a. Precision measures the relevancy provided by the users. Defined as the fraction of relevant locations among recommended locations.

$$
\mathrm{P}=\frac{\mathrm{N}_{\mathrm{rs}}}{\mathrm{N}_{\mathrm{s}}}
$$

Where $N_{r s}$ is total number of relevant locations selected, $N_{s}$ is total locations selected.

b. Recall is defined as the probability to select a relevant location to recommend.

$$
\mathrm{P}=\frac{\mathrm{N}_{\mathrm{rs}}}{\mathrm{N}_{\mathrm{r}}}
$$

Where $N_{r s}$ is total number of relevant locations selected, $N_{r}$ is total number of relevant items.

c. $F$-measure considers the harmonic mean of precision and recall

$$
\mathrm{F}=2 \frac{P \cdot R}{\mathrm{P}+\mathrm{R}}
$$

Where $P$ is precision and $R$ is recall.

\section{Related Work on Recommendation Systems}

People across the world are using smart devices and socialize with each other by linking to their social networking sites. They share location data, data of surroundings, events attended/attending and travel experiences. This data is recommended using recommendation algorithms. This section discusses various recommendation algorithms, its variants that is implemented. Also, a comparison of recommendation algorithms with the various pros and cons is being discussed. In [5] the authors 
consider the issues for location recommendations in specifically out-of-town user's preference, social influences/ connections and geographical proximity. Thereby collaborative recommendation framework-User Preference, Proximity and SocialBased Collaborative Filtering (UPS-CF) is proposed.

Out-of-town users tend to travel to places nearby and to places which is far away. The performance of recommendations for user-based collaborative filtering degrades for out-of-town places. To improve the performance locations which are far away from the current location of the target user is excluded using proximity constraints that filter out locations. The locations that are frequently visited are recommended to target users since users are likely to visit locations, which are recently visited by similar friends. The authors conclude that when users are in a town, similar users contribute more to effective recommendations, while social friends play a more vital role for users who are out-of-town.

The four variants collaborative filtering methods are:

1. Most Visited (MV) algorithm - Recommends the most visited locations based on popularity.

2. Closest Locations (CL) algorithm - Recommends the closest locations to the user's query point.

3. User-Based (U) algorithm - Recommends locations based on the recommendations of the similar users.

4. User and Proximity-Based CF (UP) - Recommends locations based on the recommendations of the similar users with the consideration of the proximity constraint. The preference derived from similar users is important for in-town users while social influences become more important for out-of-town users.

In [5], the authors propose the new approach, User and Social-Based CF (US). The UPS-CF method without consider the proximity constraint. A comparison of baseline algorithms and conventional collaborative filtering approach (and its variants) shows that UPS-CF exhibits best performance when the user is in-town, followed by US, UP, U, CL and MV for the Foursquare dataset and UP, US, U, CL, and MV for the Gowalla dataset. Since UP performs better than U and UPS performs better than US, authors conclude that removing locations farther away improves recommendation performance.

Recommendations can be achieved by friendship among the users in a recommendation system. In [6] friendship explored using two algorithms namely a) Friendship-based Bookmark-Coloring Algorithm (FBCA) b) Location-friendship Bookmark - Coloring Algorithm (LFBCA).

Book marking algorithm assumes when a node $u$ is painted, it further spreads to nearby nodes. Each node keeps $(1-a l p h a)$ portion of the paint that it receives and distributes the remaining paint to its neighbours. This transition is a recursive process which terminates if the paint to be redistributed at a node does not exceed a small constant alpha. a) Friendship-based Bookmark-Colouring Algorithm (FBCA) for all the users, personalized PageRank is calculated and further distributed to all locations visited by users. The filtering mechanism is based upon locations of user likes and does not include locations which are relatively at longer distances, away from his/her likes. And finally, $\mathrm{N}$ locations that accumulate the highest values are eventually 
recommended to the user. b) Location-Friendship Bookmark-Colouring Algorithm (LFBCA) captures both the friendship and similarity relations between users in G. Friendship edges have been replaced with similarity edges and additional similarity edges have been introduced. Each edge has a weight that quantifies how similar two users are said to be. A comparison of the proposed approach outperforms with other traditional methods.

An LBSN system usually provides support for indicating various Points of Interest (POIs), but there is no straightforward rating mechanism. Hyperlink-Induced Topic Search (HITS) is a link analysis algorithm also known as hub and authorities, wherein the authority value estimates the value of the content of a page and the hub value estimates the value of its links to other pages. In [7] proposal for a HITS based POI recommendation algorithm is discussed. It incorporates the impact of the social relationships on recommendations. POI recommendations use two measures precision and recall.

Friendship among users is one of the keys for recommendation systems. This is being further enhanced by creating groups among friends. [8] Suggests grouping among friends some pre-specified kilometers range. Also, it is suggested that the existing techniques and the services for recommendation are not efficient for on-line recommendation services. From the dataset (Foursquare), a seed is selected and user's profiles (addresses, list of friends and locations where he/she visited) are extracted using a breadth-first traversal. Each address is converted into a geographical point (i.e., latitude and longitude) from which associations are derived. Grouping of friends within $0 \sim 10 \mathrm{KM}$ forms group1, $10 \mathrm{KM} \sim 20 \mathrm{KM}$ forms group2 and so on is repeated to represent the commonly visited locations. The approach proposed is Friend-based Collaborative Filtering (FCF) based on collaborative ratings and its variant GeoMeasured FCF (GM-FCF) is based on heuristics derived from observed geospatial characteristics. A Spatio-social analysis is carried out on Foursquare data to identify unique social and spatial characteristics in such systems. The traditional approach of Collaborative Filtering is applied on the friendship.

The two variants of Collaborative filtering are proposed, Friend-based Collaborative Filtering (FCF) and Geo-Measured Friend-based Collaborative Filtering (GM-FCF). In FCF only friends are a part of collaborative filtering process. Amongst only friends the similarity weight (cosine similarity) is computed. Nonfriend users do not contribute to the recommendation hence not involved in the processing. This approach also saves time in the matrix computation and reduces noise with good precision.

GM-FCF modeling calculates similarity weight between friends and their distance by scanning friends visited locations (with its latitude and longitude). A comparison of Collaborative Filtering (CF), Friend-based Collaborative Filtering (FCF), Geo-Measured Friend-based Collaborative Filtering (GM-FCF) is done. They all use precision and recall as the metrics. Results show that FCF techniques are effective.

Presently electronic gadgets such as laptops, handheld devices such as mobiles have applications for users to collaborate and communicate with each other. In [9] location recommendation using mobiles for friends at any location is deliberated and 
named as Location-based Mobile Social Networks (LMSN). This is an instantaneous process which uses mobile social networking; here users tag locations by uploading images and other details. LMSN has the dynamic correlation characteristics of time, place and person in the real world emphasizing on user's real-time physical location proximity, offline behavior, similarity and friendship. The user's check-in dataset of Gowalla is analyzed and exposed to latent users' behavioral law in the real world.

The proposed algorithm Cos_After_SVD is used to calculate the user's behavior similarity based on the "user-time-spot" which is a three-dimensional historical check-in matrix. SVD method compares two user's behavior similarity and extracts the singular value vector of each user's check-in from the matrix. The similarity measures are Jaccard Similarity Coefficient [JSC] and average distance for friendship coefficient is used to evaluate close degree of two users. Measuring the similarity simultaneously, computes final rank scores of top- $K$ friends for target user.

One of the foremost benefit of recommendation is for improving the business of the products and the locations. [10] Presents PLUTUS framework to assist user's investment in business and maximize the expected venue's profit by recommending a set of customers to the venues (restaurant, gym, shopping mall and so on). The input parameters to the framework (taken from users) are Venue $V$, Budget/Coupon price, Number of customers (optional parameter) and the output is a set of customers who can increase the profit of the venue $V$. Each user rates the venues and these rating predictions are based on item-based collaborative filtering technique.

Two main algorithms in PLUTUS are Profit Calculation and Profit Maximization a) Profit Calculation: Calculates the total profit that a user $U$ may add to a venue $V$ considering the aspects such as social, spatial (users' geo-locations and information about users' visits to different venues) and users' opinions. Pearson Correlation is used to calculate the profit calculations. Another variation to the algorithm is Profit Calculation with Travel Penalty: Here users will limit their choice of spatial venues based on travel distance (travel penalty incurs expensive computational overhead).

b) Profit Maximization: This algorithm is used to maximize the total profit of a given venue. This algorithm has two versions, Celebrity- based Profit Maximization- with a given total marketing budget, framework recommends a set of users who can maximize the total profit of the designated venue and Coupon-based Profit Maximization - maximizes profits by distributing $\mathrm{K}$ coupons. Authors evaluate PLUTUS using foursquare real data set.

The results show that PLUTUS achieves higher estimated profit and is more efficient profit calculation than other naive marketing algorithms.

End users of any recommendation system seek at obtaining recommendation that is instantaneous indicating dynamic behavior of a user's as they move from one location to other. Location Recommendation framework with Temporal effects (LRT) is proposed in [11]. The timings of the user's profile such as the check-in and GPS data are considered to know the dynamic data. Time-dependent check-in data has higher priority than the static check-in data and thus time-dependent check-in data is given more preference. A comparison of the LRT with the User-Based Collaborative Filtering (CF), Non-negative Matrix Factorization (NMF) and Random LRT (R-LRT) is presented. The authors indicate that there is a better performance of LRT than NMF. 
Location Recommendation with various aggregation strategies such as mean, voting, maximum, sum is compared with the metrics such as precision and recall. The Foursquare data sets are used to obtain check-ins for a period of three months.

All the recommendation systems have description of the locations presented as text. With text mining technique high-quality information is derived from text. This high-quality information describes the trends, patterns and locations description. [12] Presents a methodology for social recommendations in Location Based Social Network using Text Mining techniques. The architecture methodology steps involve insertion of the user credentials such as the ID and the access code in the social network. Based on the semantic tags, each user can create their own vocabulary and explore knowledge in unexplored areas. As users give out the tags they are interested in, the social recommendation returns geo location data, which includes the latitude, longitude, and city name and so on. This also aims at recommendation based on tags using TF-IDF (Term-Frequency Inverse Document Frequency) which measures the similarity between the items. The frequency terms are presented in the form of keywords, phrases with context of the tags is measured. The recommendation is in the form of user/tag/location and item/tag/location. With the usage of collaborative tags, the cold start problem is reduced.

For the promotion of a location, the methods like Influence Maximization (IM) and Independent Cascading Diffusion Model (ICM) is used. IM identifies a set of users who can influence many other users to visit the location The ICM is based on graph models, in which any of the active nodes has a probability of changing any of its connected / neighboring inactive nodes to become active.

To determine the propagation probability of each edge the relation between users and the selected location should be detected. The location recommendation must be adaptive to the user's current location.

Any recommendation systems should pose dynamic, instant personalized recommendation. The issues such as spatial and temporal sparsity for the check-in data is addressed in Distance-based Mobility Model (DMM) [13]. DMM model utilizes random walk with restart and Pareto distribution. The algorithms proposed are the Active First and Expert First. In Active First, top- $k$ users are selected who have visited the selected location or who are near the selected location. In Expert First, a section of users who have the greatest number of social connections and those who have visited the selected location frequently are chosen. Random walk with restart property estimates probable locations where the users would stay but due to the space sparsity of check-in history these locations are few. To solve power law property of the movement, distance is explored to estimate the probability of the user moving from one location to the selected location. For the evaluation of the results Gowalla and brightkite datasets are used. The results show that DMM captures each user's mobility effectively and generates proper propagation probability. In addition, a comparison of this improved algorithm with other recommendation algorithms verifies the feasibility and effectiveness.

In Table 5 we have summarized the algorithms /Frameworks /Techniques which are covered in the LBSN recommendation system. 


\begin{tabular}{|c|c|c|}
\hline $\begin{array}{c}\text { Sl } \\
\text { No }\end{array}$ & $\begin{array}{c}\text { LBSN Concept } \\
\text { Covered }\end{array}$ & Algorithm/Framework/Technique Used \\
\hline 1 & $\begin{array}{l}\text { Recommendation } \\
\text { Systems }\end{array}$ & $\begin{aligned}> & \text { User Preference ,Proximity and Social Based } \\
& \text { Collaborative Filtering (UPS-CF) } \\
> & \text { Friendship Based Bookmark Coloring Algorithm } \\
& \text { (FBCA) } \\
> & \text { Location Friendship Bookmark Coloring } \\
& \text { Algorithm (LBCA) } \\
> & \text { Friendship Based Collaborative Filtering (FCF) } \\
> & \text { Geo measured FCF (GM-FCF) } \\
> & \text { HITS based Point of Interest Recommendation } \\
> & \text { Cos_after_SVD Algorithm } \\
> & \text { Profit Calculation and Profit Calculation with } \\
& \text { travel Penalty } \\
> & \text { Distance - Based Mobility Model (DMM) }\end{aligned}$ \\
\hline
\end{tabular}

Table 5: Summary of the LBSN surveyed papers

\section{Improving Recommendation Systems}

Based on the analysis of Section 3 we have identified few improvements like-

- Personalization, temporal changes, user's context changes, environmental context (weather, events).

- Recommendation improvements by receiving feedback/ online updates from the users directly about the accuracy of the model.

- System should provide information about the routes, places to visit based on age categories. For instance, for children - amusement parks/ zoo, and for elderly people-movies/restaurants, regional transportation and parking in and around the location.

- Explore distance between two people to recommend for instance based on nationality, occupation.

- Explore the popularity information (the historical places, historical events, famous items, food available) of a recommended location.

\section{Conclusion}

From the study the role of the Recommendation Systems in LBSN is highlighted. A brief of the Algorithms used for the recommendations is discussed.

These LBSN algorithms are being used to improve the business in terms of marketing locations and the products at recommended locations. In our further 
research we look at improving the algorithms to meet the instant and dynamic living style of users.

\section{References}

[1] Y. Zheng, "Tutorial on location-based social networks," in Proceedings of the 21 st international conference on World Wide Web, 2012, Apr 16, Vol. 12, No. 5.

[2] X. Xiao, Y. Zheng, Q. Luo and X. Xie, "Finding similar users using category-based location history," in Proceedings of the 18th SIGSPATIAL International Conference on Advances in Geographic Information Systems , 2010 Nov 2 , pp. 442-445.

[3] J. Bao, Y. Zheng and D. Wilkie, "A Survey on Recommendations in Location-based Social Networks," ACM Transaction on Intelligent Systems and Technology, 2013.

[4] R. Zafarani, M. Ali Abbasi and H. Liu, "Recommendation in

Social Media," in Social Media Mining- An Introduction, 1st ed. United States of America: Cambridge University Press, 2014, ch.9, pp. 289-317.

[5] Ference G, Ye M and Lee WC, "Location recommendation for out-of-town users in location-based social networks," in Proceedings of the 22nd ACM international conference on Information \& Knowledge Management, 2013 Oct 27 , pp. 721-726.

[6] H. Wang, M. Terrovitis and N. Mamoulis, "Location recommendation in location-based social networks using user check-in data," in Proceedings of the 21st ACM SIGSPATIAL International Conference on Advances in Geographic Information Systems, 2013.

[7] X. Long and J. Joshi, "A HITS-based POI recommendation algorithm for location-based social networks," in Proceedings of the 2013 IEEE/ACM International Conference on Advances in Social Networks Analysis and Mining, 2013 Aug 25,pp. 642-647.

[8] M. Ye, P. Yin and W. C. Lee, "Location recommendation for locationbased social networks," in Proceedings of the 18th SIGSPATIAL international conference on advances in geographic information systems ,2010 Nov 2 ,pp. 458-461.

[9] Q. Xiuquan, S. Jianchong, Z. Jinsong, X. Wangli, W. Budan, X. Sida and C. Junliang, "Recommending friends instantly in location-based mobile social networks," in China Communications, 2014 Feb, pp.109-27.

[10] M. Sarwat, A. Eldawy, M. F. Mokbel and J. Riedl, "Plutus: leveraging location-based social networks to recommend potential customers to 
venues," in Mobile Data Management (MDM), 2013 IEEE 14th International Conference on 2013 Jun 3, Vol. 1, pp. 26-35.

[11] H. Gao, J. Tang, X. Hu and H. Liu, "Exploring temporal effects for location recommendation on location-based social networks," in Proceedings of the 7th ACM conference on Recommender systems ,2013 Oct 12 , pp. 93-100.

[12] H. Wang, M. Terrovitis and N. Mamoulis, "Location recommendation in location-based social networks using user check-in data," in Proceedings of the 21st ACM SIGSPATIAL International Conference on Advances in Geographic Information Systems, 2013 Nov 5 , pp. 374-383.

[13] K. Lin, J. Wang, Z. Zhang, Y. Chen and Z. Xu, "Adaptive location recommendation algorithm based on location-based social networks," in Proceedings of the 10th International Conference in Computer Science \& Education (ICCSE), 2015 Jul 22 , pp. 137-142. 